\title{
Autorité, responsabilité, pédagogie et évaluation dans les établissements scolaires anglais
}

Authority, responsibility, teaching methods and assessment in English schools Autoridad, responsabilidad, pedagogía y evaluación en los establecimientos escolares ingleses

Peter Kelly

Traducteur : Jérôme Quintana

\section{(2) OpenEdition} Journals

Édition électronique

URL : https://journals.openedition.org/ries/5528

DOI : 10.4000/ries.5528

ISSN : 2261-4265

Éditeur

France Education international

Édition imprimée

Date de publication : 1 septembre 2016

Pagination : 115-124

ISBN : 978-2-85420-611-7

ISSN : 1254-4590

\section{Référence électronique}

Peter Kelly, «Autorité, responsabilité, pédagogie et évaluation dans les établissements scolaires anglais », Revue internationale d'éducation de Sèvres [En ligne], 72 I septembre 2016, mis en ligne le 01 septembre 2018, consulté le 01 juillet 2021. URL : http://journals.openedition.org/ries/5528 ; DOI : https://doi.org/10.4000/ries.5528 


\title{
Autorité, responsabilité, pédagogie et évaluation dans les établissements scolaires anglais*
}

\author{
Peter Kelly \\ Université de Plymouth
}

La conception utilitariste de l'éducation a débuté en Angleterre il y a plus de vingt ans, renforcée par la croyance néolibérale selon laquelle le bien-être humain ne peut progresser, dans tous les domaines, que grâce à l'individu, libre de servir ses propres intérêts à travers l'échange marchand. Wayne Au (2008) voit dans l'évaluation compétitive, qui a recours aux tests et examens à fort enjeu, l'un des principes fondamentaux au cœur de la réforme néolibérale de l'éducation. Ce type d'évaluation fournit des renseignements permettant aux parents de faire des choix d'ordre scolaire ou encore de peser sur les objectifs prioritaires de l'établissement et permet aux directeurs d'entreprises de réaliser des ajustements afin de mieux répondre à la demande du marché et d'accroître la satisfaction des parents et des élèves.

Avec un certain nombre de collègues, nous avons analysé l'histoire et le contexte de l'évaluation nationale en Angleterre (Andreasen et al., 2015), qui concerne désormais les élèves de tous les âges. En résumé, les évaluations standardisées, notamment en anglais et en mathématiques, permettent de suivre et de comparer les performances des élèves et donc des enseignants et des établissements. Des résultats plus médiocres qu'attendus pour les élèves, les enseignants et les établissements entraînent des conséquences non négligeables, pouvant porter atteinte à la réputation de certains d'entre eux et conduire à un contrôle plus sévère, souvent de la part des inspecteurs de l'Ofsted ${ }^{1}$. De mauvais résultats peuvent par ailleurs provoquer une baisse du nombre d'élèves inscrits ainsi qu'une perte de revenus pour l'établissement. Les élèves peuvent pâtir d'une mauvaise image d'eux-mêmes en tant qu'apprenants et cela peut également influencer les attentes des enseignants à leur égard lors du passage dans le secondaire. Tous ces éléments, pris dans leur ensemble, renforcent l'importance de l'impact négatif des évaluations standardisées. Selon un rapport issu d'une commission d'enquête parlementaire de la Chambre des Communes, datant de 2008, les évaluations entraînent un resserrement du curriculum autour des disciplines évaluées, accroissant de fait le temps passé à préparer les élèves à ces

\footnotetext{
* Article traduit par Jérôme Quintana.

1. L'Ofsted (Office for Standards in Education, Children's Services and Skills) est un service financé par le gouvernement britannique mais ne relevant pas de la responsabilité directe d'un ministre. Il est essentiellement chargé d'inspecter l'ensemble des établissements publics et certains établissements privés. (NdT)
} 
évaluations, favorisant un apprentissage superficiel et une rétention à court terme des connaissances, et enfin, augmentant le stress des élèves et leur démotivation. Les recherches montrent également que l'évaluation contribue à amoindrir l'importance des matières non évaluées, notamment des matières artistiques. En outre, elles indiquent que l'enseignement est davantage centré sur l'enseignant et que les ressources sont essentiellement utilisées pour venir en aide aux groupes d'élèves situés à la frontière du niveau demandé, afin de maximiser l'impact en termes de taux de réussite et de classement de l'établissement (league tables). On note aussi une hausse du nombre d'établissements qui sélectionnent et excluent les élèves pour améliorer les résultats (Stevenson et Wood, 2013).

Nous examinons dans cet article trois des principaux traits caractérisant le processus par lequel l'évaluation standardisée contribue à façonner l'enseignement en Angleterre : premièrement, la confiance accordée aux résultats des évaluations, ces résultats étant perçus comme faisant autorité dans leur manière d'apprécier les élèves, les enseignants et les établissements ; deuxièmement, l'implication des responsables des diverses modalités d'évaluation standardisée, que nous mettons en relation avec la façon dont ces modalités d'évaluation, en tant que technologies de gouvernance, peuvent formater le curriculum, la pédagogie et les perceptions subjectives des élèves ; troisièmement, les effets induits par tous ces facteurs sur la pédagogie et le vécu des élèves.

\section{Autorité et Évaluation}

On a déjà beaucoup écrit sur ce qui caractérise la "modernité tardive » (late modernity) (Bauman, 2000). Nous sommes passés, dit-on, d'une ère de stabilité économique, où l'industrie manufacturière proposait aux travailleurs un travail intéressant à vie, à une nouvelle ère où de nombreuses personnes sont concernées par l'emploi temporaire précaire et où il incombe aux employés de l'ère postindustrielle, amenés à occuper divers postes, de rester compétitifs sur le marché du travail en acceptant de se recycler en permanence. Nous sommes passés d'une société ordonnée, où chacun connaissait sa place, où le sentiment d'appartenance et le respect mutuel favorisaient la cohésion de la communauté, à une société où les travailleurs, qui ne sont désormais plus « le sel de la terre ", sont au contraire vus comme des profiteurs, des tire-au-flanc, et où les personnes issues de milieux socialement favorisés ont plus de chances d'être soupçonnées de jouir de privilèges indus que d'être considérées avec égard. Nous sommes passés d'une situation où la totale confiance que l'on accordait autrefois à certaines professions n'existe plus : d'une part, le service public en Angleterre se heurte aujourd'hui à un climat de méfiance favorisé par les articles et les reportages qui paraissent régulièrement, dénonçant les comportements scandaleux ou l'insuffisance des moyens; d'autre part, le rapport paternel qu'entretenaient les médecins, les infirmières, les agents de police, les travailleurs sociaux et les enseignants avec leur communauté, est devenu aujourd'hui en grande partie un 
rapport de professionnels à consommateurs. Nous sommes passés d'un mode d'identification collective en tant que sujets ou ressortissants d'une nation, dans une alliance forte avec des pays qui définissent clairement les limites du gouvernement, à un mode dans lequel nous formons désormais un ensemble d'individus possédant chacun des droits de citoyens mais rattachés à divers groupes d'appartenance dans des espaces sociaux marqués par la fluidité des mouvements migratoires et où, du fait de la technologie, les individus ne sont pas entravés par les limites des États-nations et les menaces en matière de sécurité et de mode de vie ne connaissent plus aucune limite. Enfin, nous sommes passés d'un mode d'appréhension du monde dans lequel celui-ci était perçu comme étant une ressource domptée par l'homme, prête à être exploitée, à un nouveau mode dans lequel les gens sont vulnérables, à la merci des éléments et jamais très loin d'une catastrophe écologique. Mises bout à bout, on peut dire de ces transformations qu'elles reflètent une crise de l'autorité de la tradition et de la certitude scientifique, qu'elles interrogent le caractère inévitable du progrès de l'humanité et qu'elles conduisent à une période où règnent davantage d'incertitude et de risque relativiste (Gray 1995).

Face à ce doute épistémique, certains ont cherché à réaffirmer l'autorité de la science, de la religion ou, sous diverses formes, d'autres visions laïques du monde, proclamant la souveraineté de leurs interprétations fondamentalistes. D'autres, plus nombreux encore, acceptent une part plus grande d'incertitude. Pour eux, il existe deux principales réponses politiques. Définissant la confiance comme le fait « de conférer celle-ci à des personnes ou à des systèmes abstraits, à partir d'un sursaut de foi faisant fi de l'ignorance ou du manque d'information » (1991), Giddens affirme que, dans ce contexte fluide, nous n'avons pas d'autre choix que de faire confiance aux institutions gouvernementales et de la société civile. Mais si pour Onora O'Neill, intervenant lors des conférences Reith de la BBC en 2002, la réponse à toute crise de l'autorité, pour les professions liées au service public, passe par la modération de leur autorité grâce à une plus forte transparence, une communication ouverte et un partenariat avec leurs clients, pour Davis (2014), en revanche, le néolibéralisme donne au marché l'autorité autrefois accordée aux individus en prônant le discours selon lequel les gens sont naturellement dotés d'un esprit de compétition. C'est pourquoi l'autorité donnée aux idées persuasives et aux positionnements de valeur, à la rigueur dans le domaine de la recherche, de l'argumentation, de la preuve, ou encore au débat et au consensus, est remplacée par la foi dans une "main invisible ». Ce changement de perspective est perceptible dans le fait que les institutions, autrefois régies par des décrets et des directives, sont aujourd'hui gouvernées par des mécanismes ou des technologies politiques qui façonnent la pratique professionnelle. Dans le domaine de l'éducation, Ball (2013) en identifie trois : marchandisation, gestion et performance.

Mais c'est là que réside une certaine tension. En Angleterre, pour mettre les choix d'établissements en concurrence, les «consommateurs d'éducation » 
demandent à être suffisamment informés pour pouvoir établir des comparaisons entre les différents prestataires de services éducatifs. Ces comparaisons sont néanmoins complexes, c'est pourquoi il faut comme intermédiaires des institutions en qui l'on peut avoir confiance, afin de rendre plus simple cette complexité au regard des consommateurs. Cela place certains organismes en position d'autorité, notamment l'agence chargée de l'inspection des établissements privés ( $\mathrm{ISI}^{2}$ ) et l'Ofsted, dont le rôle est entre autres de demander des comptes aux établissements et aux enseignants. On remarquera, avec une certaine ironie, que pour légitimer leur autorité, ces organismes se rallient aux approches inspirées de la science, mettant l'accent sur les notions d'impartialité et de détachement, en espérant que cela apporte un degré de certitude et de prévisibilité aux éventuelles complexités de la pratique sociale. Ainsi, les évaluations de l'Ofsted sont basées sur des critères clairs liés à des référentiels communs. Il est intéressant de constater que c'est dans la fiabilité de l'évaluation que l'on trouve la preuve de cette clarté des critères : deux inspecteurs, face aux mêmes données, doivent être en mesure d'attribuer la même note. Mais cette fiabilité a un coût : les principes de base qui gouvernent ces évaluations doivent être simples, sans ambiguïté et dépourvus d'aspects litigieux ; or ce sont autant de caractéristiques totalement absentes des classes et des écoles. La solution a été de passer d'une évaluation des processus à une évaluation des résultats, en partant du principe que les données peuvent "parler d'elles-mêmes ». De ce fait, les données qui influent le plus sont les performances des élèves, mesurées à partir d'évaluations standardisées réalisées dans les établissements ou d'examens standardisés sanctionnant la fin des études. À cet égard, les décideurs et les administrateurs en matière d'éducation se sont laissés séduire par les mathématiques. En effet, les données quantitatives sont perçues comme étant objectives, claires et précises, malgré les mises en garde selon lesquelles ces données ne sont utiles que dans la mesure où les hypothèses sur lesquelles elles reposent relèvent du raisonnable. Quant aux analyses statistiques, elles apportent des éléments de certitude, même si les données statistiques, comme il a été rappelé, donnent lieu à des interprétations élaborées dans des conditions d'incertitude. On comprend ainsi que, dans les établissements scolaires anglais, c'est la mesure des performances au moyen de l'évaluation standardisée qui conditionne la pratique. Comme nous l'indique Ozga (2008), nous vivons sous la tyrannie de la gouvernance par le nombre.

En 2014, avec un certain nombre de collègues, nous avons réalisé une étude de l'évaluation dans des classes du primaire en Angleterre (Andreasen et al., 2015). Dans le cadre de cette enquête, nous avons interrogé des enseignants et avons compris, à notre grande surprise, qu'ils considéraient les évaluations comme étant des outils solides et des indicateurs fiables des performances des élèves en anglais et mathématiques. Les parties qui suivent dans le présent article seront liées à certains autres résultats issus de cette enquête.

2. ISI : Independent School Inspectorate : [http://www.isi.net/about] (NdT) 


\section{RESPONSABILITÉ ET ÉVALUATION}

Jusqu'à présent, dans cet article, nous avons suggéré que le néolibéralisme est une réponse à une crise de l'autorité et que, dans l'éducation, la confiance autrefois placée dans l'autorité des enseignants a été remplacée par une croyance dans la main invisible du marché. Nous avons défendu l'idée selon laquelle les diverses formes d'évaluation nationale standardisée en Angleterre jouent un rôle important dans la gouvernance de l'éducation (Ball, 2013). Comme nous l'avons déjà laissé entendre et le démontrerons plus loin, ces diverses formes d'évaluation modifient le sens même de la pratique et façonnent de ce fait certains rapports sociaux connexes, et ce en dépit du fait que les praticiens, tels que les enseignants du primaire interrogés dans le cadre de cette étude, déclarent souvent n'accorder à ces évaluations qu'une importance modérée. Mais examinons tout d'abord le processus par lequel les modes d'évaluation façonnent le curriculum, la pédagogie et les perceptions subjectives des élèves. Bien qu'il s'agisse d'un processus complexe, l'un des facteurs importants est de déterminer qui, parmi ceux chargés de rendre des comptes, sont les mieux placés pour influencer ce processus d'évaluation et par conséquent de déterminer qui pourrait tirer partir ou au contraire pâtir des résultats de ces évaluations.

On accorde tellement de poids aux résultats des évaluations et des examens nationaux obtenus par les établissements que, selon un enseignant interrogé, " ces résultats représentent tout ce pour quoi l'établissement travaille, plus que toute autre chose ». Les résultats sont utilisés pour catégoriser et comparer les établissements et entrevoir leurs progrès d'année en année. Ils ont un impact sur la fréquence et l'intensité des inspections et des interventions de l'Ofsted et, in fine, sur le fait de poursuivre ou de modifier l'axe de l'établissement en termes de développement, de gestion et de personnel. En outre, comme le pointe un autre enseignant, les gouverneurs, les collègues, les parents et les futurs parents évaluent les établissements et les enseignants en fonction de leurs résultats publiés et des commentaires qu'en font les médias locaux. Cela joue sur la popularité d'un établissement, sur son mode de recrutement des élèves et sur la réputation individuelle des enseignants. C'est la raison pour laquelle les équipes de direction des établissements déploient une énergie considérable non seulement pour tenter d'obtenir les meilleurs résultats possibles, mais aussi pour anticiper la façon dont l'Ofsted interprétera ces résultats, principale préoccupation des équipes étant donné l'importance des évaluations de l'Ofsted tant pour les établissements que pour chacun des enseignants qui y travaillent.

Les enseignants sont évalués ouvertement à partir des résultats des élèves. Notamment lors de la phase de préparation intensive aux épreuves et examens nationaux, ils bénéficient du statut spécial que leur confère l'importance donnée à la bonne préparation des élèves, mais ils se retrouvent par ailleurs dans 
une position délicate car ils risquent de voir leur réputation ternie en cas de mauvais résultats. Les propos d'un enseignant du primaire sont à ce propos intéressants :

«En tant qu'enseignant en Year 6 (dernière année du primaire), je ressens une forte pression car je ne peux pas me permettre de lâcher prise, vu les attentes des parents et de l'école. »

Néanmoins, pour éviter de trop dépendre d'un seul enseignant, dans de nombreux établissements anglais, on répartit la responsabilité de la préparation des élèves et de l'organisation des évaluations sur l'ensemble d'une équipe d'enseignants et d'assistants pédagogiques.

Ainsi, les résultats obtenus aux diverses épreuves et aux divers examens ont autant d'importance pour l'équipe de direction que pour les enseignants, dont les postes et la réputation dépendent des performances réalisées, sans parler de l'importance de ces dernières aux yeux de nombreux parents et élèves convaincus que leur avenir en dépend. En conséquence, toutes les parties concernées croient de bon cœur aux formules de bon sens que leur adresse l'Ofsted et pensent que leurs intérêts sont directement liés aux résultats. Rien ne montre que le processus d'évaluation est perçu comme étant valable en tant que tel par tous ceux qui s'investissent un tant soit peu dans cette question des résultats. En effet, il est possible que la place prépondérante accordée à l'évaluation externe et aux performances en Angleterre ne permette pas d'apprécier de quelque façon que ce soit ce que peut apporter l'évaluation standardisée aux enfants sur le plan de leur formation ou de leur développement individuel.

Dans ce contexte, les individus ou les groupes les mieux placés pour s'assurer que les évaluations nationales concourent à la défense de leurs intérêts s'emploient à poursuivre cet objectif. Les équipes de direction comme les enseignants exploitent et s'approprient les rapports d'inspection individualisés de l'Ofsted, fondés sur les données, pour être sûrs de tirer le meilleur parti des évaluations nationales tout en s'attirant la complaisance des parents et des élèves, dont l'influence est moindre. Ainsi, les enseignants veillent à identifier des connaissances importantes, des méthodes pédagogiques efficaces et des individus les plus à risque. En s'appuyant par exemple sur des notions telles que les "niveaux-frontières » (grade-borderlines), on identifie des groupes-cibles ou groupes d'appui composés d'élèves dont le travail, selon les enseignants, se situe précisément à la limite ou à la frontière et qui courent le risque de se trouver en deçà des attentes à l'issue des épreuves d'évaluation nationale. Et prédomine un enseignement direct où les élèves subissent un contrôle très fort. Conférer aux seuls enseignants le pouvoir de décider permet à ces derniers de démontrer leur valeur et leur grande expertise. Certains s'avèrent plus doués dans ce domaine que d'autres, certains élèves répondent mieux que d'autres à cette approche, qui permet d'identifier certaines pratiques de classe comme étant efficaces. 


\section{Pédagogie et évaluation}

Pour Bernstein (1996), la pédagogie peut s'analyser en fonction de ce que l'on peut définir comme étant des connaissances importantes, en fonction aussi de la façon dont l'apprentissage s'opère et de ce qui constitue une preuve légitime de cet apprentissage. S'intéressant tout particulièrement à l'organisation de l'enseignement, à sa gestion et à son discours, il oppose deux modèles. Dans le modèle pédagogique fondé sur les performances, les enseignants tentent de manière explicite de contrôler ce que les élèves apprennent et de quelle façon. Le modèle fondé sur les compétences, en revanche, est moins formel dans la mesure où les enseignants répondent aux besoins des élèves en partageant, semble-t-il, ce contrôle et en se préoccupant des résultats à la fois tangibles et intangibles de l'apprentissage. Ce sont des modèles que l'on peut considérer comme étant des concepts-idées qui, servant de supports de comparaison, permettent de mettre de l'ordre dans le chaos du monde. Si on les utilise comme éléments de comparaison pour examiner le système scolaire anglais, on observe que celui-ci a évolué, passant des compétences, qui dominèrent le mouvement progressiste, notamment dans les établissements primaires de la fin des années 1960 et des années 1970, aux performances.

Les recherches menées conjointement avec Andreasen et al., font apparaître clairement le fait que l'évaluation nationale et les technologies d'analyse et de transmission des données qui lui sont associées ont constitué le principal moteur de l'actuel modèle pédagogique fondé sur les performances. Le mode d'évaluation actuellement en place en Angleterre, au sein d'une culture fortement individualisée, fondée sur l'instrumentalisation et orientée vers le résultat, a conduit les établissements à axer leur enseignement presque entièrement sur les connaissances à évaluer selon ces modalités d'évaluation, notamment durant la dernière année de l'enseignement primaire et lors des deux années qui mènent à l'examen du GCSE [le General Certificate of Secondary Education, que les élèves passent à l'âge de 16 ans]. Par ailleurs, dans les écoles primaires, pour prendre leur exemple, le suivi détaillé et régulier des résultats des élèves prévaut encore, ainsi que l'enseignement ciblé des stratégies à mettre en œuvre pour les tests d'évaluation, même si ces pratiques ont très peu à voir avec l'amélioration des compétences des enfants en lecture, expression écrite et mathématiques. Ce qui prédomine, c'est une vision de l'enseignement où l'on entraîne les élèves à acquérir certaines compétences utiles. Cela est en partie dû, comme nous l'avons indiqué, au fait que ces tests visent moins à évaluer les élèves qu'à servir de principal instrument de mesure de la responsabilité des établissements et des enseignants pour qui l'enjeu est grand et qui, par conséquent, cherchent à obtenir une certaine constance dans les performances des élèves aux tests d'évaluation et à les optimiser grâce à un entraînement intense et complet. C'est ainsi que nous avons observé des enseignants se focaliser sur des gains à court terme dans les tests d'évaluation durant la dernière année du primaire, enseignant 
essentiellement des stratégies aux élèves et tirant ainsi le meilleur parti des ressources dont ils disposent, plutôt que d'essayer de s'intéresser aux facteurs qui permettraient, par exemple en lecture, d'améliorer la maîtrise de l'exercice et la compréhension de l'écrit et d'exposer les élèves à un plus grand nombre de textes issus de genres différents; ce sont là des objectifs qui prennent du temps. Les nouvelles technologies permettent de suivre de très près les progrès des élèves et cet entraînement peut donc être ciblé de manière très précise.

Wayne Au décrit «le rapport entre [...] les changements induits par l'évaluation dans les pratiques de classe et la reproduction [...] des inégalités dans l'éducation » (Au 2008), laissant entendre que les tests ou épreuves à fort enjeu fonctionnent comme relais dans la reproduction des rapports sociaux dominants dans l'éducation. Sur cette question, il s'inspire de la description faite par Bernstein du procédé pédagogique (1996) qui consiste à proposer que les connaissances de certains groupes sociaux, ainsi que leur conscience, soient considérées comme relevant surtout du bon sens, délimitant ainsi le pensable de l'impensable et marginalisant ceux dont les normes se situent en dehors de ces paramètres. Il affirme que certaines façons bien déterminées de parvenir à la connaissance sont transposées dans des curriculums et des approches pédagogiques via des processus de recontexualisation. Cela permet ainsi à certains élèves et à certains enseignants, ainsi qu'à certaines pratiques de classe, d'être identifiés et valorisés par rapport à d'autres. Pour Au, les tests standardisés à fort enjeu sont ainsi l'expression de formes dominantes de connaissances et de conscience, appliquées ensuite à divers groupes sociaux (Bernstein, 1996). De façon plus précise, et d'après les résultats de nos enquêtes, les tests : (a) définissent ce qui relève des connaissances légitimes à l'école; (b) exercent un contrôle considérable sur la manière dont les enseignants présentent les connaissances et le contenu, souvent proche de celle utilisée dans les tests ; enfin, (c) ils contribuent à renforcer le contrôle des pratiques pédagogiques des enseignants et à conduire ces derniers vers des pratiques davantage centrées sur l'enseignant, afin de mieux s'adapter aux types de connaissances et de contenus qu'exigent les tests.

Du fait des pratiques identifiées plus haut, les tests d'évaluation façonnent les perceptions subjectives des élèves, à mesure que ceux-ci prennent conscience de leurs relatives forces et faiblesses, au point que certains d'entre eux se définissent eux-mêmes ainsi que leurs camarades sur le plan scolaire en ces termes. Les élèves les moins performants évoquent, d'une part, leur plus grande prédisposition à dépendre de l'aide de leurs enseignants et, d'autre part, leur passivité dans le processus d'apprentissage, autorisant volontiers les enseignants à prendre en charge leur apprentissage. Cette dépendance et cette passivité les conduisent à être complices de leur propre marginalisation à travers le processus d'évaluation mis en place. Les élèves les plus performants, de leur côté, ont toute confiance dans leurs chances de réussite, qu'ils anticipent avec délectation grâce à leur parfaite maîtrise des normes de l'évaluation. Par ailleurs, les 
élèves en Angleterre acceptent le fait que certains domaines de connaissances (notamment ceux faisant l'objet d'une évaluation) sont plus importants que d'autres (par exemple, l'art, les lettres, l'éducation physique et tout ce qui relève de l'apprentissage informel comme les représentations théâtrales à l'école ou les sorties scolaires) et attachent de l'importance au travail scolaire durant leur dernière année et notamment aux examens qui bénéficient d'un statut plus élevé que le travail demandé lors des années précédentes : la préparation aux examens exige en effet un gros effort collectif et une plus grande maturité individuelle. Ici, l'intérêt des connaissances réside entièrement dans leur utilité plutôt que dans leur valeur intrinsèque.

À travers ces processus, les élèves deviennent des biens marchands : leurs performances sont le fruit d'une discipline qui passe par l'entraînement et la répétition et, en échange, elles servent à améliorer la qualité de leur établissement. Les élèves sont juges de leur propre valeur mais ils sont aussi jugés par les autres, non seulement par rapport à leur aptitude dans telle ou telle matière et par rapport au fait que celle-ci soit considérée comme étant sérieuse ou plutôt futile, mais également en termes de leur importance pour la réussite de leur établissement. Ces éléments mis bout à bout permettent en partie de comprendre le développement des perceptions subjectives néolibérales des élèves (Keddie, 2016). Ces perceptions subjectives occupent une place plus importante car les tests d'évaluation, marquant de fait la fin des études à l'école primaire en Angleterre, sont devenus un rite de passage pour les jeunes.

\section{UN AUTRE AVENIR POSSIBLE}

Pour revenir à O'Neill, il nous est possible d'entrevoir un autre avenir - un avenir autrefois décrit par Isaiah Berlin (1978). Dans cet avenir démocratique, on accepte l'idée que les connaissances sont faillibles et on cherche par conséquent à modérer l'autorité experte des spécialistes de l'éducation et des administrateurs scolaires grâce à une plus grande transparence et à une communication plus ouverte. Mais il n'appartient pas ici au marché d'appréhender l'ensemble des choix rationnels effectués par les individus : l'éducation est un bien social et individuel porteur de valeurs et de ce fait, les décisions relatives aux établissements scolaires sont politiques, non pas seulement économiques. Toutefois, les établissements scolaires offrent un service à des individus et à des groupes possédant de nombreux points de vue différents sur les caractéristiques d'une bonne société ; la gouvernance de ces établissements doit ainsi impliquer un partenariat entre les diverses parties prenantes du processus éducatif, et plus particulièrement les élèves, les parents et les professionnels de l'éducation. En rassemblant les forces, il est possible de trouver des solutions temporaires et partielles à des problèmes sociaux complexes, à travers des débats qui prennent au sérieux les divers points de vue des différentes catégories de personnes et cherchent à créer un esprit d'entente réciproque afin de mieux intégrer certains 
positionnements de valeur parfois difficilement réconciliables. L'évaluation standardisée peut encore jouer un rôle dans cet avenir-là, mais celui d'un indicateur imparfait parmi tant d'autres, utilisé de manière critique au même titre que d'autres approches pour évaluer l'apprentissage et le développement. Par ailleurs, en élargissement la base à partir de laquelle ces évaluations sont réalisées, nous pourrions, au lieu de transformer en biens marchands des élèves dont on dédaigne la profondeur et la complexité, célébrer au contraire les diverses formes de réussite de tous nos enfants à mesure qu'ils évoluent dans le système scolaire jusqu'à l'âge adulte.

Le jeu en vaut assurément la chandelle.

\section{BIBLIOGRAPHIE}

ANDREASEN K., KELLY P., KOUSHOL K., McNESS E., YDESEN C. (2015) : «Standardised testing in compulsory schooling in England and Denmark: A comparative study and analysis ", Bildung und Erziehung, 68(3), 329-348.

AU W. (2008) : «Devising inequality: A Bernsteinian analysis of high-stakes testing and social reproduction in education, British Journal of Sociology of Education, 29(6), 639-651.

BALL S. (2013): The education debate (second edition), Bristol, Policy Press.

BAUMAN Z. (2000) : Liquid modernity, Cambridge, Polity Press.

BERLIN I. (1978) : Russian thinkers, London, Penguin.

BERNSTEIN B. (1996): Pedagogy, symbolic control and identity: theory, research, critique, London, Taylor Francis.

GIDDENS A. (1991) : Modernity and self-identity, Cambridge, Polity Press.

GRAY J. (1995) : Enlightenment's wake: politics and culture at the close of the modern age, London, Routledge.

HOUSE OF COMMONS (2008): Testing and assessment: third report of session 2007-08, [en ligne] [goo.gl/sY3Qwk] [consulté le $1^{\mathrm{er}}$ mai 2016].

KEDDIE A. (2016) : "Children of the market: performativity, neoliberal responsibilisation and the construction of student identities ", Oxford Review of Education, 42(1), 108-122.

OZGA J. (2008) : "Governing knowledge: Research steering and research quality ", European Educational Research Journal, 7(3), 261-272.

STEVENSON H., WOOD P. (2013) : " Markets, managerialism and teachers' work: the invisible hand of high-stakes testing in England ", The International Education Journal Comparative Perspectives, 12(2), 42-61. 